\section{Detection of iron deficiency in children with Down syndrome}

To the editor:

Recently, we read the report by Hart and colleagues with great interest. ${ }^{1}$ In this study, authors aimed to evaluate the utility of biochemical and red blood cell indices for detecting iron deficiency (ID) and ID anemia (IDA) in Down syndrome (DS). They showed that the combination of red cell distribution width (RDW) with ferritin or transferrin saturation (TS) percentage led to $100 \%$ sensitivity, and RDW combined with ferritin showed the highest discriminability for ID/IDA. They concluded that a complete blood count and ferritin should be obtained routinely for children over 1 year old with DS rather than hemoglobin alone for detection of ID. Although the results were interesting, some points should be noted.

First, definition of ID solely based on serum ferritin or transferrin saturation might erroneously estimate the prevalence. As pointed out in previous reports, serum ferritin is an acute-phase protein that reflects body iron stores. Multiple confounding situations may artificially increase its concentrations, such as inflammation, infection, oxidative stress, liver disease, autoimmune disease, and renal failure. ${ }^{2,3}$ These disorders might raise serum ferritin levels up to more than $1500 \mu \mathrm{g} / \mathrm{L}^{4}$ Moreover, metabolic situations and innutrition may decrease serum transferrin concentrations, resulting in misleadingly low elevations of transferrin saturation. ${ }^{5}$ These details should be considered in the inclusion criteria. Although bone marrow biopsy remains the gold standard investigation of ID/IDA, it is usually avoided because of its invasiveness. Other markers could be added to the definition of ID/IDA, such as soluble transferrin receptor (sTfR), which is increased in tissue iron deficiency and not sensitive to inflammation. ${ }^{5,6}$ Other reports also indicate that the sTfR/log ferritin ratio (TfR-F index) was highly correlated with body iron stores and indicative of subclinical ID. ${ }^{6}$

Second, the study revealed that RDW combined with ferritin showed the highest discriminability for ID/IDA. However, numerous confounding factors could affect RDW levels. As a marker of red blood cells' heterogeneity, RDW was implicated as a valuable parameter in multiple disorders, including inflammatory diseases and cardiovascular diseases. However, increasing reports have demonstrated numerous confounding factors that raised or decreased RDW levels. For example, infectious diseases, autoimmune diseases, diabetes, and metabolic syndrome. ${ }^{7,8}$ Furthermore, drugs (antibiotics and anticoagulant) and antianemia therapy (erythropoietin and chalybeate) at baseline or follow-up might also affect RDW levels. Measurement details (tube with anticoagulant, delay time for blood collection and measuring) could change RDW levels or reference range.," 10 Details of these would help to demonstrate the clinical role of RDW in ID/DIA definition.

\section{DISCLOSURE}

The authors declare no conflicts of interest.

Publisher's note Springer Nature remains neutral with regard to jurisdictional claims in published maps and institutional affiliations.

\section{Wei-yun Zhang, $M M^{1}$, Ying-ying Liu, $M M^{1}$ and Da-xiong Zeng, $M D \mathbb{B}^{1}$}

${ }^{1}$ Department of Pulmonary and Critical Care Medicine, The First Affiliated Hospital of Soochow University, Suzhou, P. R. China. Correspondence: Da-xiong Zeng (zengdaxiong@suda.edu.cn)

\section{REFERENCES}

1. Hart SJ, Zimmerman K, Linardic CM, et al. Detection of iron deficiency in children with Down syndrome. Genet Med. 2019 Aug 16; https://doi.org/ 10.1038/s41436-019-0637-4 [Epub ahead of print].

2. Pasricha SR, Flecknoe-Brown SC, Allen KJ, et al. Diagnosis and management of iron deficiency anaemia: a clinical update. Med J Aust. 2010;193:525-532.

3. Lopez A, Cacoub P, Macdougall IC, Peyrin-Biroulet L. Iron deficiency anaemia. Lancet. 2016;387:907-916.

4. Hearnshaw S, Thompson NP, McGill A. The epidemiology of hyperferritinaemia. World J Gastroenterol. 2006;12:5866-5869.

5. Wish JB. Assessing iron status: beyond serum ferritin and transferrin saturation. Clin J Am Soc Nephrol. 2006;1:S4-S8.

6. Camaschella C. New insights into iron deficiency and iron deficiency anemia. Blood Rev. 2017;31:225-233.

7. Tonelli M, Sacks F, Arnold M, et al. Relation between red blood cell distribution width and cardiovascular event rate in people with coronary disease. Circulation. 2008;117:163-168.

8. Lippi G, Cervellin G, Sanchis-Gomar F. Red blood cell distribution width and cardiovascular disorders. Does it really matter which comes first, the chicken or the egg? Int J Cardiol. 2016;206:129-130.

9. Salvagno GL, Sanchis-Gomar F, Picanza A, Lippi G. Red blood cell distribution width: a simple parameter with multiple clinical applications. Crit Rev Clin Lab Sci. 2015;52:86-105.

10. Lippi G, Pavesi F, Bardi M, Pipitone S. Lack of harmonization of red blood cell distribution width (RDW). Evaluation of four hematological analyzers. Clin Biochem. 2014;47:1100-1103.

Advance online publication 12 November 2019. doi:10.1038/s41436-019-0699-3 